\title{
Las prácticas inclusivas en los sistemas complejos
}

\author{
Inclusive practices in complex systems
}

Raquel Susana Sandrone ${ }^{1}$ Carolina del Valle Erramouspe ${ }^{2}$ Stella Maris Adrover ${ }^{3}$

Resumen: En la complejidad de la realidad en que estamos insertos, la diversidad de sujetos que conviven en las escuelas amerita una reinterpretación de las prácticas de enseñanza encaminadas a la inclusión de todos y cada uno de esos estudiantes. En este ensayo, intentamos reflexionar sobre las prácticas inclusivas desde las teorías complejas y sus principios. Concluimos que la clave está en el respeto hacia la contradicción que se genera entre pensar en la inclusión de todos y la selectividad de cada uno.

Palabras clave: diferenciación, sistema educativo, complejo, práctica pedagógica, teoría del aprendizaje.

\footnotetext{
${ }^{1}$ Especialista en Gestión Directiva. Especialista en Política Socioeducativa. Especialista en Matemática para primer ciclo. Licenciada en Educación General Básica. Inspectora Técnica en la Zona Escolar 2350, Ministerio de Educación de la provincia de Córdoba, Argentina. Correo electrónico: rasandrone67@gmail.com.

2 Especialista en Inclusión Educativa de Personas con Discapacidad. Licenciada en Ciencias de la Educación. Licenciada en Gestión de la Educación Especial. Profesora en Educación de Sordos. Profesora en Deficientes Mentales. Integrante del Equipo Técnico de la Dirección General de Educación Especial y Hospitalaria de la provincia de Córdoba, Argentina. Correo electrónico: erramouspecaro@gmail.com.

${ }^{3}$ Especialista en Multimpedidos. Licenciada en Educación. Psicopedagoga. Directora General de Educación Primaria, Ministerio de Educación de la provincia de Córdoba, Argentina. Correo electrónico: stellamarisadrover@yahoo.com.ar.
}

Diálogos Pedagágicas. ISSN en línea: 2524-9274.

Año XVIII, No 35, abril - septiembre 2020. Pág. 37-46.

DOI: http://dx.doi.org/10.22529/dp.2020.18(35)03 / Recibido: 25-08-2019 / Aprobado: 7-02-2020

(c) (i) () Artículo publicado bajo Licencia Creative Commons Atribución-NoComercial-SinDerivar. By NC No (c) Universidad Católica de Córdoba. 
Abstract: In the complexity of the reality in which we are inserted, the diversity of subjects coexisting in schools merits a reinterpretation of teaching practices aimed at the inclusion of each and every one of those students.

In this essay, we try to think about inclusive practices from complex theories and their principles. We conclude that respect towards contradiction is key, which occurs when thinking about everybody's inclusion and each other's selectivity.

Key words: differentiation, educational system, complex, teaching practice, learning theory.

\section{Introducción}

Hoy, los que transitamos el siglo XXI, nos encontramos con una realidad incierta, cambiante, diversa e intercomunicada, compleja, que traspasa las puertas y se instala en las aulas de todas las escuelas, lo cual desafía -de esta manera- a las políticas públicas que intentan responder al principio de la inclusión educativa y sus prácticas.

¿Qué factores intervienen o hacen a las prácticas incluyentes en las escuelas? La escuela hoy puede reconocer la diversidad y la multiculturalidad. Pero en la práctica, ese conocimiento no se trasunta en acciones heterogéneas que consideran las singularidades y los contextos. Esta situación produce angustias, frustraciones y dudas por no poder dar respuestas a las demandas del escenario educativo.

Internacionalmente, la problemática de la exclusión y de la inequidad se plasma en la Declaración de Incheon, según el Marco de Acción Educación 2030, aprobado en noviembre de 2015 (Unesco, 2015), que ofrece orientación a los gobiernos y a sus socios para convertir los compromisos en acción. Su artículo 7 declara:

La inclusión y la equidad en la educación y a través de ella son la piedra angular de una agenda de la educación transformadora, y por consiguiente nos comprometemos a hacer frente a todas las formas de exclusión y marginación, las disparidades y las desigualdades en el acceso, la participación y los resultados de aprendizaje. Ninguna meta educativa debería considerarse lograda a menos que se haya logrado para todos. Por lo tanto, nos comprometemos a realizar los cambios necesarios en las políticas de educación y a centrar nuestros esfuerzos en los más desfavorecidos, especialmente aquellos con discapacidad, para velar por que nadie se quede atrás. (p. 7)

La inclusión educativa es una innovadora e inexcusable visión de la educación basada en la diversidad, la cual implica la aceptación y valoración de las diferencias y el reconocimiento a todos como sujetos de derecho, pero consideramos imprescindible, en estos momentos, poner en duda lo que sabemos y proyectarlo a lo que hacemos. 
Si bien el acceso a la escuela para todos fue un paso fundamental para asegurar el derecho a la educación, no se acaba allí la cuestión para garantizar la inclusión. Flavia Terigi (2011) habla de la "conformación de circuitos de escolarización diferenciados" que vulneran las trayectorias educativas de los estudiantes dejándolos excluidos.

Considerar la igualdad como punto de partida lleva implícita la generación de un clima de cuidado y la confianza en las posibilidades de aprender del otro. Como educadores, es nuestra obligación brindar a nuestros estudiantes las herramientas para entender el mundo, reflexionar e interpretar la realidad en que se desenvuelven para que, a través de la confianza, amparo y cuidado, en el sentido atribuido por Perla Zelmanovich (2002), sean capaces de transformarla e idear su propio proyecto de vida.

Este ensayo intenta una desnaturalización de las prácticas inclusivas que ya son parte de la realidad de muchas aulas desde el enfoque epistemológico de los sistemas complejos.

\section{Desarrollo}

\section{I- De los sistemas complejos}

El enfoque del pensamiento complejo engloba la idea de que cualquier elemento del mundo no es considerado un objeto aislado, sino que forma parte de un sistema mayor que lo contiene, por lo que se encuentra en constante interacción con otros elementos del sistema, así como con el sistema completo. De este modo, la realidad cobra sentido en el concepto de sistemas complejos. Morín (2004) nos presenta lo real como algo muy poco conocido. Nos habla de que, para diagnosticar lo real en la actualidad, tenemos que rechazar el realismo trivial, según el cual hay que adaptarse a lo inmediato. Es decir, lo real es un hervidero de posibilidades y nunca sabremos qué resultará de ello. Desde una mirada bio-antropo-sociológi$\mathrm{ca}$, debemos entenderlo como una totalidad integrada, un modelo de interconectividad, no lineal y autorregulable.

El desafío de la complejidad consiste, precisamente, en el reconocimiento de las tramas o redes de relaciones y la imposibilidad humana de agotarlas en el conocimiento. La relación, en una sociedad compleja, abarca los ámbitos biológico, económico, espiritual, político, cultural e histórico, entre otros. La complejidad no desecha ningún paradigma, sino que lo retoma desde la complementariedad de miradas y se sitúa como un sistema abierto. Su objetivo principal, según Duván Marín Gallego (2012), es contextualizar y globalizar y, a la vez, recoger lo que arroja la incertidumbre del mundo globalizado. Para lograrlo, existen tres teorías:

1) La teoría de la información es una herramienta que trata básicamente de cómo recibir un mensaje codificado como señal a través de un canal ruidoso (Capra, 2006, p. 83). El término tiene un sentido altamente técnico muy diferente del uso corriente, que trata la incertidumbre, la sorpresa y lo inesperado, al mismo tiempo que entra en un universo donde hay, también, orden (redundancia) y 
desorden (ruido) y extrae de ahí algo nuevo. Es decir, la información deviene organizadora (programadora) de una máquina cibernética.

2) La cibernética "es una teoría de los sistemas de control basada en la comunicación (transferencia de información) entre sistema y medio circundante, y dentro del sistema, y en el control (retroalimentación) del funcionamiento del sistema en consideración al medio" (Von Bertalanffy, 2007, p. 20). Es la teoría de las máquinas autónomas, autoconducidas y autorreguladas. La idea de retroacción o retroalimentación rompe con el principio de causalidad lineal al introducir el principio de bucle causal o bucle de retroalimentación, como una disposición circular de elementos conectados causalmente en la que una causa inicial se propaga alrededor de los eslabones sucesivos del bucle, de tal modo que cada elemento tiene un efecto sobre el siguiente, hasta que el último retroalimenta el efecto sobre el primer eslabón en que se inició el proceso (Capra, 2006, p. 75).

3) La teoría de los sistemas se refiere a las bases fundamentales de un pensamiento de la organización; por lo tanto, la primera lección de esta teoría es la siguiente:

Que el todo es más que la suma de sus partes. Significa esto, que existen cualidades emergentes, es decir, que nacen de la organización de un todo y que pueden retroactuar sobre sus partes (Bunge, 2004). Además, considera que el todo es igualmente menos que la suma de las partes, puesto que ellas pueden tener cualidades inhibidas por la organización del conjunto (Gonzáles, 1997). (Marín Gallego, 2012, p. 162)

Según el mismo autor, también existen tres principios que complementan las teorías antes mencionadas:

- El principio dialógico, entendido como la asociación complementaria de dos opuestos que se repelen y se necesitan para ser, existir o funcionar organizadamente.

- El principio de recursividad organizacional, que describe la relación del todo y las partes entendiendo a los organismos como causas y efectos de su propia existencia, como productos y productores de sus propias realidades.

- El principio hologramático, que cobra significado en la presencia no solo de las partes en el todo, sino -también- la presencia del todo en cada una de sus partes, tal como sucede en una figura holográfica.

El estudio de lo complejo ha impactado, también, en el ámbito más directo de las interacciones de los seres humanos: la educación, la interpretación de la sociedad, la política, y la comprensión del momento actual que vive la humanidad. El problema de la complejidad ha pasado a ser el problema de la vida y el vivir, el problema de la construcción del futuro y la búsqueda de soluciones a los problemas contemporáneos.

Por lo tanto, como educadores, consideramos a las prácticas inclusivas como uno de los problemas contemporáneos más recurrentes y significativos. Intentaremos avanzar en su análisis a través de la interpretación de las teorías y principios que sustentan el enfoque de los sistemas complejos. 


\section{II- De las prácticas inclusivas}

El desafío que enfrentan los sistemas educativos actuales es responder con equidad y justicia al dilema de las diferencias en la educación escolar. No se trata de indicar, sugerir estrategias o técnicas específicas ni de imponer cambios organizativos en las escuelas, sino de poner atención a los procesos de aprendizaje social de los actores en el escenario educativo, dentro de contextos particulares. La colaboración y el empleo de las evidencias como un medio para estimular la innovación y la mejora educativa son consideradas las dos estrategias clave para orientar estos procesos en una dirección más incluyente. En este contexto, la indagación y reflexión sobre la práctica juega un papel importante para desarrollar el "liderazgo distribuido" que precisa el proceso de inclusión, pues -como sugiere Copland (2003)- dicha indagación colaborativa puede ser el "motor" para activar la participación.

Dentro de las escuelas y los sistemas educativos

El punto de partida de este interminable proceso debe ser siempre el de involucrar a todos los profesionales que trabajan alrededor de la tarea educativa, en todos los niveles, ampliando su capacidad de reflexionar e imaginar lo que podría ser alcanzado, así como aumentando su sentido ético y la responsabilidad en el logro de tal fin. (Booth \& Escudero, 2006 en Echeíta \& Ainscow, 2011)

Se hace imprescindible, en este punto, poner en tensión las concepciones desde un posicionamiento ético político e implícito que impregna la práctica docente.

Por lo anteriormente expuesto y posicionados desde la complejidad al educar en y para la diversidad, el abordaje educativo en el aula no puede reducirse ni centrarse en las dificultades o diferencias de los sujetos con respecto a la norma o situación como único factor, sino en el conjunto de recursos educativos que la escuela debe prever y proveer para dar una respuesta social y educativa a todos los estudiantes.

Blanco (1999, en Ávila Durán \& Esquivel, 2009) plantea: "Dar respuesta a la diversidad significa romper con el esquema tradicional en el que todos los niños hacen lo mismo, en el mismo momento, de la misma forma y con los mismos materiales" (p. 79). La meta por lograr, por parte de los docentes, es cómo pueden ellos organizar la enseñanza para todos los estudiantes, sin dejar de lado las necesidades particulares de cada uno.

Necesitamos comenzar a reinterpretar los sentidos que se entretejen en el devenir de las prácticas educativas. Lo que observamos en las escuelas es que se desarrollan prácticas estancas y atomizadas con actividades planificadas de manera desarticulada, disociadas de la concepción de aprendizaje. Podríamos volver a pensar: ¿En qué espacios enseñamos? ¿Podemos provocar la ruptura del aula estándar, del currículum único, de métodos uniformes que vienen a testimoniar nuestras dificultades para el tratamiento de lo diverso?

Para este fin, es importante interpelar formatos instalados, naturalizados, para posibilitar la revisión de prácticas y construir formas innovadoras, una valora- 
ción de cada espacio escolar para conocer a cada uno de los estudiantes, al alojarlo en sus particulares modos de estar y de aprender y ofrecer una propuesta flexible que contemple las diversas formas de aprender de todos y cada uno de ellos.

Diferentes corrientes pedagógicas proponen un cambio de mirada sobre las didácticas empleadas por los docentes en las aulas, las complejizan y -de esta manera- dan la posibilidad de abordar la diversidad de los estudiantes a los que acompañan. Una de estas corrientes es la pedagogía crítica, cuyo enfoque didáctico fomenta el aprendizaje cooperativo y colaborativo y piensa en los rasgos singulares de cada uno de los estudiantes, sus modos de aprender, sus contextos y su experiencia, entre otros aspectos. En definitiva, una didáctica que permite a cada sujeto el desarrollo de su integralidad para enlazarlo a un proyecto colectivo.

Este posicionamiento implica un docente interesado en la tarea que realiza y con disposición para llevar adelante prácticas significativas, creativas e innovadoras, en contextos complejos. Estas prácticas buscan brindar propuestas con plena conciencia de la diversidad a la que entiende y asume como desafío. Su tarea será capaz de seleccionar y organizar contenidos y diseñar propuestas de enseñanza, de manera tal que, por diferentes caminos, los estudiantes puedan lograr diversas metas.

El respeto por la diversidad en la escuela es complejo, no tiene respuestas claras ni definitivas, sino que requiere respuestas contextualizadas y la reflexión permanente acerca de los efectos que nuestras decisiones e intervenciones tienen sobre nuestros estudiantes.

\section{III- Las teorías complejas y las prácticas inclusivas}

Como ya lo hemos mencionado con anterioridad, el objetivo del enfoque de los sistemas complejos es contextualizar y globalizar para evitar las incertidumbres de la globalización. Para lograrlo, existen teorías que lo permiten. Este pensamiento nos lleva a preguntarnos: ¿Cómo se interpretan las prácticas inclusivas en las teorías complejas?

La teoría de la información nos permite plantearnos las prácticas inclusivas desde la mirada de todos los modelos pedagógicos, de la diversidad curricular, del contexto de aprendizaje, de la accesibilidad material y simbólica y de las necesidades educativas de todos y cada uno de nuestros estudiantes. También nos permite generar una nueva mirada que no se trate sobre los problemas de aprendizaje y se focalice en los problemas de la enseñanza.

Deberíamos preguntarnos ¿Qué es la diversidad para nosotros? ¿Quiénes son esos, nuestros estudiantes? ¿Qué estamos pensando en relación con el otro? ¿Quién es ese otro? ¿Cómo me posiciono en relación con sus diferencias? ¿Considero la diversidad como un valor educativo?

Entonces, cuando obtengamos algunas de estas respuestas, estaremos habilitados para ensayar trayectorias múltiples y diversas de aprendizajes interaccionistas y colaborativos. Intentaremos llevar al aula la mayor cantidad posible 
de propuestas, recursos y estrategias para que cada uno pueda construir el aprendizaje escolar, pensando en una escuela amplia, plural, democrática. Contaremos con la posibilidad de generar las condiciones de enseñanza desde la multiplicidad de estrategias curriculares y lograr una diversidad tan expandida que permita involucrar a todos los estudiantes desde su lugar particular.

Los docentes -desde esta perspectiva- tienen estrategias pedagógicas para posibilitar una educación inclusiva de calidad: el diseño universal del aprendizaje es una de ellas. La Convención de las Personas con Discapacidad (ONU, 2006) establece que:

Por "diseño universal" se entenderá el diseño de productos, entornos, programas y servicios que puedan utilizar todas las personas, en la mayor medida posible, sin necesidad de adaptación ni diseño especializado. El "diseño universal" no excluirá las ayudas técnicas para grupos particulares de personas con discapacidad, cuando se necesiten. (p. 5)

El diseño universal aplicado a la enseñanza y al aprendizaje escolar implica replantear las viejas planificaciones y estrategias implementadas para todos de igual manera, de modo uniforme, donde el estudiante con discapacidad recibe una "adecuación curricular" diferente al resto. Podemos pensar estrategias de enseñanza que planteen distintas maneras de organizar los espacios, los tiempos, los agrupamientos de los alumnos, los canales de comunicación y el uso de los recursos.

Construir una práctica que contemple la mirada desde el diseño universal implica la organización de una propuesta compleja, situada y flexible, pensada para todos y cada uno de los estudiantes, que abarque lo colectivo desde la singularidad, los modos de aprender, los intereses y las capacidades.

También consideramos que es a través de la teoría cibernética que contamos con la posibilidad de permitir, en cada práctica inclusiva, la puesta en marcha de procesos de metacognición, entendidos estos como la acción y el efecto de razonar sobre el propio razonamiento, de desarrollar conciencia y control sobre los procesos de pensamiento y aprendizaje, lo que permite entender la manera en que se piensa y aprende y aplicar ese conocimiento sobre estos procesos para obtener mejores resultados.

Poder saber la forma en que cada uno es capaz de aprender nos permite autorregular nuestras estrategias a fin de aprender mejor. En un aula inclusiva, este autoaprendizaje, compartido con el docente, permitirá la selección más apropiada de las condiciones de enseñanza para cada uno de los estudiantes para habilitar así el desarrollo máximo de sus potencialidades para que puedan participar activamente en la cultura. Nuestra propuesta debe estar atravesada por la cultura. Podríamos preguntarnos: ¿Qué de lo cultural, que deberíamos enseñar, está presente en la vida de nuestros estudiantes?

Por su parte, la teoría de los sistemas nos lleva a revisar y repensar los modos de atención al derecho social a la educación en aulas heterogéneas. Entender a todos y a cada uno de nuestros estudiantes como sujetos de derecho es com- 
prender la necesidad de atención a sus particularidades a lo largo de su trayectoria escolar. Esto implica una mirada profunda a las representaciones de normalidad y anormalidad, lo común y lo diferente, el sentido de la escuela y la corresponsabilidad que todos tenemos. Implica, también, la mirada institucional de cada situación. La trayectoria del estudiante la construimos todos.

En la mirada institucional del cuidado de las trayectorias escolares, es necesario adoptar una mirada interinstitucional que permita asegurar entornos de accesibilidad y participación. Accesibilidad entendida como un concepto abarcativo: accesibilidad física, simbólica, emocional y material.

Es aquí que la mirada compleja, necesaria para que suceda la inclusión en cada práctica, depende de entender que el todo es más que la suma de cada una de sus partes.

Pensando desde otro sentido la teoría de los sistemas, abrimos la posibilidad de habilitar las prácticas inclusivas desde desafíos significativos para cada uno de los estudiantes, que sean representativos de su contexto de procedencia real y que les permitan una mirada integrada a los diferentes campos del conocimiento. El formato de secuencia didáctica y de proyecto son los más apropiados para esta mirada sistémica de los contenidos y aprendizajes.

\section{IV-Principios del enfoque de los sistemas complejos y las prácticas inclusivas}

Los principios que complementan las teorías del enfoque de los sistemas complejos también son referentes válidos para ampliar las posibilidades de interpretación de las prácticas inclusivas.

El principio dialógico nos permitiría poner en foco los procesos de inclusión y, a la vez, los de selectividad necesarios para garantizar trayectorias exitosas a todos nuestros estudiantes. Se trata de ver el aula desde el lugar del deber de enseñar todo a todos, pero desde las posibilidades que cada uno ha desarrollado, desde esa particularidad que cada uno manifiesta y, a su vez, saber que todos son capaces, que todos pueden.

El principio de recursividad organizacional, por su parte, nos muestra otra arista en la mirada compleja a las prácticas inclusivas. La diversidad étnica, lingüística, poblacional, de clase social, subjetiva (de formas de pensar), familiar, de discapacidad, entre otras, produce una riqueza inigualable si trabajamos desde el paradigma de la complementariedad. En este sentido, lo construido en cada aula tendrá que ver con el aporte particular que realiza cada uno de sus integrantes, que conforma así una cultura propia en las formas de ser y actuar. Pero a la vez, esta construcción colectiva que se ha logrado como cultura del aula irá modificando ciertos rasgos de las formas personales e individuales de cada estudiante. Además, la reflexión que hacemos desde el principio hologramático nos permite redimensionar la realidad social entendiendo la presencia no solo de las partes en el todo, sino -también- la presencia del todo en cada una de sus partes. 
Y por qué a la realidad social, porque consideramos que a la realidad social la conforma cada una de las microrrealidades existentes en cada espacio social, pero que, a su vez, en cada una de estas realidades y, para el caso que analizamos, cada una de nuestras prácticas áulicas encierra la realidad social existente en el mundo presente.

Es por ello que consideramos la diversidad del aula como causa y efecto de la diversidad originada en la globalización y la necesidad de contextualizarla y entendemos que, en cada contexto, también encontraremos esa diversidad que caracteriza a la globalización.

\section{A modo de cierre...}

Avanzar en el análisis de las prácticas inclusivas a través de la interpretación de las teorías y principios que sustentan el enfoque de los sistemas complejos reclama un debate en el interior de las escuelas para construir un escenario didáctico que sea accesible para todos nuestros estudiantes.

Existirían -en lo educativo- dos aspectos que son importantes de observar y considerar. Por un lado, la idea de inclusión, su definición a nivel de sistema y, también, a nivel de escuelas y docentes. Por otro lado, los modos de ver y medir el rendimiento educativo y las creencias que circulan en torno a la calidad y éxito educativo.

Nuestro trabajo como docentes y el de los responsables en torno a la educación es el de poder, en primera instancia, definir la inclusión, ya que, desde ella, se orientan las acciones y las líneas de política educativa con coherencia. Cabe decir, también, que -al hablar de educación inclusiva- es necesario tener en cuenta las condiciones generales del contexto social, político y pedagógico en el que los procesos y las prácticas inclusivas se llevan a cabo haciendo foco en las características locales, culturales e históricas de cada escenario.

Las prácticas inclusivas permiten trabajar la diversidad de nuestras aulas, las cuales se entienden como continuidades de lo que sucede en el mundo actual. Es necesario que, a través de ellas, se respete el derecho de todos los estudiantes desde la atención a la diversidad curricular, a las trayectorias múltiples y diversas, a la implementación de secuencias o proyectos que partan de un desafío real y contextualizado.

Para ello, debemos tener en cuenta un amplio desarrollo de la metacognición como proceso de autorregulación del propio aprendizaje y como generador de nuevas condiciones de enseñanzas, sin perder de vista la corresponsabilidad que nos compete como institución.

La clave está en el respeto a la contradicción que se genera entre pensar en la inclusión de todos y la selectividad de cada uno. Nos posibilita poner a trabajar lo colectivo y lo singular, interpretar tramas particulares y grupales. Consideramos que ese entretejido transforma a todos y a cada uno. 
La construcción de prácticas inclusivas desde este posicionamiento nos remite a pensar en términos de complejidad, nos convoca a considerar a la institución como un todo que construye intervenciones socioeducativas que atienden a temáticas relacionadas con el aprendizaje y la enseñanza en un contexto y en configuraciones ambientales destinadas a favorecer encuentros, lo que da lugar a experiencias subjetivantes.

\section{Referencias bibliográficas}

Ávila, A. \& Esquivel, V. (2009). Educación Inclusiva en nuestras aulas. Colección Pedagógica Formación Inicial de Docentes Centroamericanos de Educación Básica. Costa Rica: Editorama.

Capra, F. (2006). La trama de la vida. Barcelona: Anagrama.

Copland, P. (2003). The Bioethical Structure of a Human Being. Recuperado el 16 de marzo de 2020, de https://doi.org/10.1111/1468-5930.00241.

Echeíta, G. \& Ainscow, M. (2011). La educación inclusiva como derecho: marco de referencia y pautas de acción para el desarrollo de una revolución pendiente. Tejuelo. Revista de Didáctica de la Lengua y la Literatura, 12, 26-46.

Marín Gallego, J. D. (2012). La investigación en educación y pedagogía. Sus fundamentos epistemológicos y metodológicos. Bogotá: USTA.

Morín, E. (2004). Introducción al pensamiento complejo. México, D. F.: Gedisa.

ONU (2006). Convención sobre los Derechos de las Personas con Discapacidad. Recuperado el 28 de julio de 2019, de https://www.un.org/esa/socdev/enable/ documents/tccconvs.pdf.

Terigi, F. (2011). Las cronologías de aprendizaje: un concepto para pensar las trayectorias escolares. Pensar la Escuela 2. Buenos Aires: Ministerio de Educación de la Nación.

Unesco (2015). Declaración de Incheon: Educación 2030: Hacia una educación inclusiva y equitativa de calidad y un aprendizaje a lo largo de la vida para todos. Recuperado el 20 de junio de 2019, de https://unesdoc.unesco.org/ark:/48223/ pf0000245656_spa

Von Bertalanffy, L. (2007). Teoría General de los Sistemas: Fundamentos, desarrollo, aplicaciones. Traducido por Juan Almela. Buenos Aires: Fondo de Cultura Económica.

Zelmanovich, P. (2002). Infancia, escuela y subjetividad. Clase virtual No 9 presentada en el Diploma Superior en Currículum y prácticas escolares en contexto. Buenos Aires: FLACSO. 